\title{
EL EFECTO DEL COMPORTAMIENTO EXPORTADOR SOBRE EL COMPROMISO DEL PROPIETARIO- GERENTE CON LA INNOVACIÓN EN COLOMBIA: INTERPRETACIONES BAJO EL ENFOQUE DE APRENDIZAJE*
}

\author{
Izaias Martins ${ }^{* *}$ \\ Eduardo Gómez-Araujo *** \\ Yancy Vaillant ${ }^{* * * *}$
}

\footnotetext{
doi:10.11144/Javeriana.cao27-49.ecec. Este artículo es producto de una investigación de los autores. El artículo se recibió el 12/06/2014 y se aprobó el 01/12/2014. Sugerencia de citación: Martins, I., Gómez-Araujo, E. y Vaillant, Y. (2014). El efecto del comportamiento exportador sobre el compromiso del propietario-gerente con la innovación en Colombia: interpretaciones bajo el enfoque de aprendizaje. Cuadernos de Administración, 27 (49), 135-153. Los autores agradecen a sus universidades y sus respectivos grupos de investigación el apoyo recibido para la realización del estudio. Izaias Martins agradece especialmente a la Dirección de investigación de la Universidad EAFIT por el apoyo recibido para la escritura y divulgación de los resultados de esta investigación. Por su parte, Eduardo GómezAraujo agradece la financiación recibida del Fondo de Ciencia, Tecnología e Innovación del Sistema General de Regalías en el marco del proyecto de investigación Diamante Caribe y Santanderes liderado por la Universidad del Norte, Barranquilla, Colombia (código del proyecto 2014000100012). http://dx.doi.org/10.11144/Javeriana.cao2749.ecec

** Ph.D. en Entrepreneurship and Business Management de la Universidad Autónoma de Barcelona, Barcelona, España. Profesor Asistente del Departamento de Contaduría Pública de la Universidad EAFIT, Medellín, Colombia. Correo electrónico: imartins@eafit.edu.co

*** Ph.D. en Entrepreneurship and Business Management de la Universidad Autónoma de Barcelona, Barcelona, España. Profesor Asistente del Departamento de Finanzas y Organizaciones de la Universidad del Norte Barranquilla, Colombia. Correo electrónico: araujoe@uninorte.edu.co

**** Ph.D. en Entrepreneurship and Business Management de la Universidad Autónoma de Barcelona, Barcelona, España. Profesor Asociado de ESC Rennes School of Business, Rennes, Francia. Correo electrónico: Yancy.vaillant@ esc-rennes.fr
} 
El efecto del comportamiento exportador sobre el compromiso del propietario-gerente con la innovación en Colombia: interpretaciones bajo el enfoque de aprendizaje

\section{RESUMEN}

El objetivo del artículo es investigar el efecto del comportamiento exportador de la empresa sobre el compromiso del propietario-gerente con la innovación. Para ello, se considera la teoría de aprendizaje como marco de referencia. El estudio utiliza información a partir de la encuesta de población adulta del GEM Colombia 2010 y 2011. Para verificar las hipótesis se utilizaron modelos de regresión logística considerando el efecto marginal. Los resultados indican que la propensión exportadora aumenta la probabilidad de compromiso del propietario-gerente con la innovación, corroborando la idea del aprendizaje organizacional a partir de la presencia en los mercados internacionales. Sin embargo, no se pudo confirmar el mismo efecto positivo cuando la variable explicativa ha sido la intensidad exportadora.

Palabras clave: Exportaciones, innovación, Teoría de aprendizaje.

Clasificación JEL: D21, F10, M16
The effect of export behavior on ownermanager's commitment to innovation in Colombia: insights from learning approach

\section{Abstract}

The main objective of this paper is to analyze the effect of the firm's exportbehavior on the owner-manager's commitment to innovation. The principles of the Learning Theory are used to guide the framework of the study. To test the proposed hypotheses, a logistic regression model considering marginal effects is applied to a dataset from the Global Entrepreneurship Monitor's Adult Population Survey of Colombia for 2010 and 2011. The study's results conclude that the exporting tendency increases the probability of the owner-manager's commitment towards innovation, confirming the idea of organizational learning from the presence in the international marketplace. However, we were no able to verify the same positive effect when the explanatory variable has been the export intensity.

Key words: Exports, innovation, Learning Theory. JEL Classification: D21, F10, M16

\begin{abstract}
RESUMO
$\mathrm{O}$ efeito do

O objetivo deste artigo é pesquisar o efeito do comportamento exportador da empresa sobre o compromisso do proprietário-gerente com a inovação. Para comportamento exportador sobre o compromisso do proprietário-gerente com a inovação na Colômbia: interpretações sob o enfoque de aprendizagem isso, considera-se a teoria de aprendizagem como referencial. Este estudo utiliza informação a partir da pesquisa de população adulta do GEM Colômbia 2010 e 2011. Modelos de regressão logística que consideram o efeito marginal foram utilizados para verificar as hipóteses propostas. Os resultados indicam que a propensão exportadora aumenta a probabilidade de compromisso do proprietário-gerente com a inovação, o que corrobora a ideia da aprendizagem organizacional a partir da presença nos mercados internacionais. Contudo, não se pode confirmar o mesmo efeito positivo quando a variável explicativa foi a intensidade exportadora.
\end{abstract}

Palavras-chave: Exportações, inovação, teoria da aprendizagem. JEL Classificação: D21, F10, M16 


\section{Introducción}

A pesar de que Latinoamérica y el Caribe (LAC) es una región de emprendedores, que ha disfrutado de una actividad exportadora considerable durante la última década, presenta debilidades que afectan el crecimiento de las empresas de la región, y una de ellas es precisamente la falta de innovación. El nivel reducido de inversión, tanto pública como privada, en I+D y las pocas patentes registradas por empresas de LAC son síntomas de la baja intensidad y calidad de la innovación en la región (Lederman, Messina, Pienknagura y Rigolini, 2014). El resultado de ello es un conjunto de empresas que ofrecen productos y servicios con poco énfasis en la innovación y con discreto valor agregado lo que les dificulta competir globalmente (Amorós y Cristi, 2008).

Por esta razón, tiene relevancia analizar, desde la perspectiva del propietario-gerente latinoamericano, la relación entre el comportamiento exportador y la probabilidad de compromiso del propietario-gerente con la innovación. Dentro de LAC, tomar a un país como Colombia para dicho estudio, resulta interesante porque es la cuarta mayor economía y el tercer país más poblado de la región (Banco Mundial, 2014). Además, Colombia es el país que lidera la propensión a emprender, según informes en América Latina (Varela, Moreno y Soler, 2013). Los continuos esfuerzos para mejorar el entorno para los negocios resulta promisorio considerando las oportunidades de mercado con alrededor de 20 tratados de libre comercio suscritos por el país. Como resultado del buen comportamiento de su economía, en el año 2013 Colombia fue invitado oficialmente a ser miembro de la Organización para la Cooperación y el Desarrollo Económico, OCDE, entidad que afirma que el país sigue teniendo unas perspectivas sólidas de crecimiento a corto plazo gracias a sus políticas macroeconómicas, al apogeo de los productos básicos y a las mejoras en temas de seguridad. Finalmente, Colombia como caso de estudio puede aportar evidencias relevantes por tratarse de una economía en proceso ascendiente y con proyecciones de crecimiento por encima del promedio de LAC (OCDE, 2013).

En el ámbito académico, durante la última década surgieron investigaciones sobre la relación entre innovación y comportamiento exportador (ej. Caldera, 2010; Cassiman y Golovko, 2011; Denicolai, Zucchella y Strange, 2014; Kirbach y Schmiedeberg, 2008; Lachenmaier y Wobmann, 2006), e incluso, la complementariedad que presenta la innovación y el desempeño exportador sobre el crecimiento de la empresa (Golovko y Valentine, 2011). Sin embargo, el objeto de estudio en la mayor parte de estas investigaciones es la empresa y no el propietario-gerente de la organización. Además, mayoritariamente son evidencias empíricas de característica transversal (considerando un año en el análisis). En cambio hay relativamente pocos estudios sobre los antecedentes y las fuerzas motrices de la capacidad innovadora (Hult, Hurley y Knight, 2004).

De conformidad con la Teoría de aprendizaje se propone un análisis considerando una relación positiva derivada de la presencia de la empresa en mercados internacionales, a par- 
tir de su comportamiento exportador, sobre el compromiso del propietario-gerente con la innovación. Por consiguiente, esta investigación quiere ofrecer evidencia empírica que ayude a entender dicho fenómeno en la región, y que contribuya tanto a la academia como a los responsables de las políticas públicas (Cohen y Levinthal, 1990; Lane y Lubatkin, 1998).

El presente artículo está organizado de la siguiente manera: la primera sección presenta el marco teórico, con una breve inspección de estudios previos y las hipótesis propuestas; la segunda sección, especifica la metodología de investigación y describe la base de datos utilizada; la tercera sección presenta la estimación de los resultados; y para terminar se provee un apartado de discusión de los resultados y las conclusiones del estudio.

\section{Marco teórico, estudios previos e hipótesis}

Por un lado, existe una creciente aceptación sobre la existencia de un efecto positivo de la innovación sobre la propensión a incursionar en mercados internacionales (Caldera, 2010; Cassiman y Golovko, 2011; Wakelin, 1998), así como sobre el volumen de exportaciones (Cassiman y Martínez-Ros, 2007; López Rodríguez y García Rodríguez, 2005). Por otro lado, recientemente la literatura sobre negocios internacionales ha destacado el análisis de la relación inversa o incluso la reciprocidad entre la innovación y el comportamiento exportador (Filipescu, Prashantham, Rialp y Rialp, 2013; MonrealPérez, Aragón-Sánchez y Sánchez-Marín, 2012). La perspectiva de aprendizaje me- diante la actividad exportadora, de la expresión anglosajona learning-by-exporting (Salomon y Shaver, 2005; Salomon y Jin, 2008), puede servir como marco principal para explicar cómo las empresas aprenden de los mercados extranjeros y aplican estos conocimientos en su favor.

\subsection{Exportaciones e innovación a la luz de la Teoría de aprendizaje}

La teoría de aprendizaje sugiere que las empresas aprenden más cuando dedican significantes esfuerzos en procesar nuevos conocimientos adquiridos fuera de la organización (Autio, Sapienza y Almeida, 2000; Cohen y Levinthal, 1990; Zahra y George, 2002). La habilidad para identificar y absorber nuevos conocimientos en entornos externos es un componente clave para la generación de conocimiento y para el desarrollo de la innovación (Lane y Lubatkin, 1998; Zahra y George, 2002). Además, según la visión basada en el conocimiento, la capacidad innovadora es un proceso intensivo de información y conocimiento que genera un importante conjunto de recursos intangibles (Gassmann y Keupp, 2007; Prashantham, 2005). Por ello, con el fin de ser creativos y generar productos o servicios singulares, los propietarios-gerentes necesitan acceder a la información y obtenerla de tantas fuentes como les sea posible, incluso de otros países (Kafouros, Buckley, Sharp y Wang, 2008). El conocimiento sobre los mercados extranjeros, así como la eficiencia por medio de la cual dicho conocimiento es aprendido y usado, será un determinante del éxito en empresas emprendedoras (Autio et al., 2000). Así, en las empresas con propensión 
innovadora, los propietarios-gerentes desarrollan conocimientos únicos y capacidades que hacen que el proceso de desarrollo de nuevos productos o servicios sea fluido y dinámico logrando una expansión constante del mercado (Eiriz, Faria y Barbosa, 2013). De igual manera, los propietarios-gerentes aprovechan el impulso tecnológico para innovar en la creación y el desarrollo de nuevos productos, así como en la adaptación de productos ya existentes (López Rodríguez y García Rodríguez, 2005).

Para muchas empresas, la internacionalización no es sólo una idea que llega tarde o temprano, sino una táctica esencial en sus estrategias (Sapienza, De Clercq y Sandberg, 2005). Así, la internacionalización altera inevitablemente el foco de atención estratégica de una empresa (Ocasio, 1997). Las empresas internacionalizadas a menudo pueden acceder a diversos insumos basados en el conocimiento que no están disponibles en el mercado interno, y este conocimiento puede regresar a la empresa en forma de aprendizaje, fomentando así una mayor innovación (Salomon y Shaver, 2005).

De acuerdo con Anand y Khanna (2000) y Chetty y Wilson (2003), los contactos externos pueden ayudar a la organización a desarrollar nuevas capacidades y proveer acceso a ciertos recursos, sean tangibles o intensivos en conocimiento. Esto es, las organizaciones pueden aprender de los mercados externos $\mathrm{y}$ de su presencia en el exterior (Sapienza et al., 2005). Además, las empresas internacionalizadas pueden incrementar sus redes de contacto alrededor del mundo para acceder a profesionales calificados y lograr experti- cia técnica que impulse nuevas tecnologías (Kafouros et al., 2008).

A pesar de no tratarse de un estudio longitudinal, esta investigación asume en conformidad con hallazgos previos (ej. Eriksson, Johanson, Majkgard y Sharma, 1997; Monreal-Pérez et al., 2012; Sapienza et al., 2005) que los propietarios-gerentes $\mathrm{y}$, consecuentemente las organizaciones, pueden incrementar su compromiso hacia la innovación por medio del mejoramiento del proceso de acumulación de conocimiento y del aprendizaje organizacional (Kafouros et al., 2008). Así, los propietarios-gerentes de las empresas aprenden directamente de sus experiencias en los mercados internacionales e, indirectamente, con la observación de compañías extranjeras (Johanson y Vahlne, 1991).

La discusión previa sugiere dos ideas para captar los efectos del aprendizaje a través de las exportaciones, teniendo en cuenta los datos y la información disponible para el análisis. La primera idea está enfocada en la propensión a exportar. Las empresas exportadoras, debido a su presencia internacional, tienen mayor probabilidad de aprender de otros mercados y a aplicar estos conocimientos en la generación de ventajas competitivas sostenibles basadas en la singularidad e innovación de productos o servicios, así como, el uso de tecnología de proceso reciente e innovadora (Filipescu, Rialp y Rialp, 2009; Prashantham, 2005). Así, la Hipótesis 1 propone una relación positiva entre la propensión a exportar y el compromiso del propietario-gerente con la innovación. 
Hla. En las empresas con propensión exportadora, será mayor la probabilidad de compromiso del propietario-gerente con la innovación en productos o servicios.

H1b. En las empresas con propensión exportadora, será mayor la probabilidad de compromiso del propietario-gerente con la innovación en tecnologías de proceso.

La segunda idea está más enfocada en el volumen de ventas al exterior para captar los efectos del aprendizaje a través de las exportaciones, lo que podría caracterizar mayor experiencia en lugar de considerar únicamente la participación o no en otros mercados (Fernandes e Isgut, 2005). La teoría de aprendizaje sugiere que mientras más intenso y repetitivo sea el proceso, mayor será la adquisición de conocimiento (Sapienza et al., 2005). Cuando una organización dedica más recursos y obtiene más ventas al exterior, la intensidad de su esfuerzo de aprendizaje en estos contextos también puede incrementar (Ocasio, 1997). Por lo tanto, la Hipótesis 2 propone una relación positiva entre la intensidad exportadora y el compromiso del propietario-gerente con la innovación.

H2a. En las empresas con intensidad exportadora media/alta, será mayor la probabilidad de compromiso del propietario-gerente con la innovación en productos o servicios.

H2b. En las empresas con intensidad exportadora media/alta, será mayor la probabilidad de compromiso del propietario-gerente con la innovación en tecnologías de proceso.

\section{Metodología de investigación}

\subsection{Base de datos}

Los datos usados en este estudio fueron tomados de la encuesta de población adulta (APS) del Global Entrepreneurship Monitor (GEM) Colombia, considerando información de los años 2010 y 2011. GEM monitorea la actividad emprendedora mundial y la relaciona con el crecimiento económico, con una amplia cobertura mundial y con metodología de recolección de datos unificada (Reynolds, Bosma, Autio, Hunt, De Bono, Servais, López-García y Chin, 2005).

En vías de garantizar la validez de los resultados de la encuesta, la metodología GEM está compuesta por tres fuentes de información: la población adulta entre 18 y 64 años de edad, encuesta a expertos nacionales con la intención de validar datos sobre la innovación y la actividad emprendedora y fuentes secundarias relacionadas con variables socioeconómicas.

En este estudio, la unidad de análisis son los individuos que están al frente de alguna organización ya constituida (considerando únicamente aquellos individuos que poseían o dirigían un negocio cuando se hizo la encuesta). La muestra final incluye respuestas para 1050 individuos en el año 2010 y 782 individuos en el año 2011. Las características de la muestra son similares en los dos años incluidos en el análisis. Es decir, de los individuos encuestados aproximadamente el $35 \%$ (en el año 2010 ) y el $30 \%$ (en el año 2011) son mujeres. Según la edad, en ambos años aproximadamente el 30\% están en el 
grupo etario entre 18 y 34 años, mientras casi el $70 \%$ tienen edades entre 35 y 64 años. Ver características de la muestra en el cuadro 1.

\subsection{Variables}

Propensión a exportar: variable binaria usada con la intención de identificar compañías con cierta experiencia vendiendo en mercados internacionales. Esta variable mide si la empresa tiene un comportamiento enfocado a la exportación o no. Si el 1\% o más de las ventas son al exterior, esta variable asume el valor uno (1). Por otro lado, si la empresa no tiene ventas al exterior, esta variable asume valor nulo (0). González-Pernía y PeñaLegazkue (2011) utilizan una medida similar para definir la propensión exportadora.

Intensidad exportadora: esta variable corresponde a la tasa de ventas en el exterior dividida entre el total de ventas en un periodo específico (Pan y Chi, 1999). De hecho, de acuerdo con Katsikeas, Leonidou y Morgan (2000), este es el criterio principal para medir el desempeño y la participación de una organización en el ámbito internacional. Por tanto, creamos una variable binaria que asume valor nulo (0) si las tasas de exportación están entre el 1\% y el 25\% (baja intensidad)

\section{Cuadro 1. Características de la muestra}

\begin{tabular}{|c|c|c|c|c|c|}
\hline Característica & & \multicolumn{2}{|c|}{2010} & \multicolumn{2}{|c|}{2011} \\
\hline Individuos & & $\mathrm{N}$ & $\%$ & $\mathrm{~N}$ & $\%$ \\
\hline Genero & $\begin{array}{l}\text { Hombres } \\
\text { Mujeres }\end{array}$ & $\begin{array}{l}680 \\
370\end{array}$ & $\begin{array}{l}64,8 \\
35,2\end{array}$ & $\begin{array}{l}540 \\
242\end{array}$ & $\begin{array}{l}69,1 \\
30,9\end{array}$ \\
\hline Edad & $\begin{array}{l}\text { De } 18 \text { a } 34 \text { años } \\
\text { De } 35 \text { a } 64 \text { años }\end{array}$ & $\begin{array}{l}337 \\
713\end{array}$ & $\begin{array}{l}32,1 \\
67,9\end{array}$ & $\begin{array}{l}263 \\
519\end{array}$ & $\begin{array}{l}33,6 \\
66,4\end{array}$ \\
\hline \multicolumn{6}{|l|}{ Compromiso con la innovación } \\
\hline $\begin{array}{l}\text { Comprometidos con la innovación en productos } \\
\text { o servicios }\end{array}$ & $\begin{array}{l}\text { Sí } \\
\text { No }\end{array}$ & $\begin{array}{l}476 \\
574\end{array}$ & $\begin{array}{l}45,3 \\
54,7\end{array}$ & $\begin{array}{l}616 \\
166\end{array}$ & $\begin{array}{l}78,8 \\
21,2\end{array}$ \\
\hline $\begin{array}{l}\text { Comprometidos con el uso de tecnologías de pro- } \\
\text { ceso recientes }\end{array}$ & $\begin{array}{l}\text { Sí } \\
\text { No }\end{array}$ & $\begin{array}{l}330 \\
720\end{array}$ & $\begin{array}{l}31,4 \\
68,6\end{array}$ & $\begin{array}{l}277 \\
505\end{array}$ & $\begin{array}{l}35,4 \\
64,6\end{array}$ \\
\hline \multicolumn{6}{|l|}{ Características específicas de la empresa } \\
\hline Propensión a exportar & $\begin{array}{l}\text { Exporta } \\
\text { No exporta }\end{array}$ & $\begin{array}{l}408 \\
642\end{array}$ & $\begin{array}{l}38,9 \\
61,1\end{array}$ & $\begin{array}{l}465 \\
317\end{array}$ & $\begin{array}{l}59,5 \\
49,5\end{array}$ \\
\hline Intensidad exportadora & $\begin{array}{l}\text { Baja } \\
\text { Media/alta }\end{array}$ & $\begin{array}{r}322 \\
86\end{array}$ & $\begin{array}{l}78,9 \\
21,1\end{array}$ & $\begin{array}{l}364 \\
101\end{array}$ & $\begin{array}{l}78,3 \\
21,7\end{array}$ \\
\hline Tamaño de la empresa & $\begin{array}{l}\text { 1-5 empleados } \\
6-19 \text { empleados } \\
20 \text { o más }\end{array}$ & $\begin{array}{r}863 \\
136 \\
51\end{array}$ & $\begin{array}{r}82,2 \\
13,0 \\
4,9\end{array}$ & $\begin{array}{r}597 \\
153 \\
32\end{array}$ & $\begin{array}{r}76,3 \\
19,6 \\
4,1\end{array}$ \\
\hline $\begin{array}{l}\text { Tiempo de constitución } \\
\text { Babybusiness/establecidas }\end{array}$ & $\begin{array}{l}\text { Hasta } 42 \text { meses } \\
\text { Más de } 42 \text { meses }\end{array}$ & $\begin{array}{l}469 \\
581\end{array}$ & $\begin{array}{l}44,7 \\
55,3\end{array}$ & $\begin{array}{l}418 \\
364\end{array}$ & $\begin{array}{l}53,45 \\
46,55\end{array}$ \\
\hline
\end{tabular}

Fuente: elaboración propia. 
y el valor uno (1) si la tasa de exportación es mayor o igual a 26\% (medio/alta). Nuestra clasificación está fundamentada en Acs y Amorós (2008), quienes capturaron la "orientación empresarial exportadora" considerando la importancia de una "tasa relativamente alta de mercado externo". El propósito es generar una clasificación que apunte a identificar las firmas con una proporción creciente y significativa de sus ingresos derivados de ventas al exterior y cómo ello puede afectar el compromiso con la innovación según los propietarios-gerentes de la organización.

Compromiso con la innovación: este constructo es parte de la información disponible en la encuesta GEM y sus dimensiones se refieren a la postura del propietario-gerente de una organización frente al reto de desarrollar soluciones internas que sean creativas y novedosas u ofertas externas con estas mismas características (Reynolds et al., 2005). Las preguntas que se refieren al compromiso con la innovación indican los grados de esfuerzo del propietario-gerente de la empresa en un intento para que los clientes potenciales consideren sus productos o servicios novedosos, y las tecnologías y procedimientos requeridos por estos productos o servicios son nuevas y/o están siendo actualizadas constantemente. Por tanto, de las preguntas relativas al compromiso con la innovación tenemos: respecto a la innovación en productos o servicios, esta asume el valor uno (1) para los casos en que el propietario-gerente afirma un compromiso hacia la innovación y valor nulo (0) cuando no se evidencia el compromiso. Para la tecnología de procesos utilizada en la generación del producto o servicio ofrecido por la empresa, asume valor uno (1) para los casos en que el propietario-gerente afirma un compromiso con la utilización de tecnologías de menos de cinco año de creadas (nuevas) y valor nulo (0) cuando el compromiso no es evidenciado y las tecnologías utilizadas han sido creadas hace más de cinco años (obsoleta). El cuadro 2 presenta las preguntas utilizadas para medir el compromiso del propietario-gerente con la innovación.

\section{Cuadro 2. Preguntas para la captura del compromiso con la innovación}

\begin{tabular}{|l|l|}
\hline $\begin{array}{l}\text { Compromiso con } \\
\text { la innovación en } \\
\text { productos o ser- } \\
\text { vicios }\end{array}$ & $\begin{array}{l}\text { ¿Usted está comprometido y hace } \\
\text { todo el esfuerzo para que su po- } \\
\text { tencial cliente considere los pro- } \\
\text { ductos y/o servicios nuevos y poco } \\
\text { familiares? }\end{array}$ \\
\hline $\begin{array}{l}\text { Compromiso con } \\
\text { el uso de tecnolo- } \\
\text { gías de proceso } \\
\text { nuevas }\end{array}$ & $\begin{array}{l}\text { ¿Usted está comprometido con } \\
\text { recientes (menos de un año y entre } \\
\text { uno y cinco años), siempre que es- } \\
\text { tas sean requeridas en el desarrollo } \\
\text { del producto o servicio ofrecido? }\end{array}$ \\
\hline
\end{tabular}

Fuente: elaboración propia a partir de Reynolds et al. (2005).

\subsection{Variables de control}

Género: en el caso de Colombia, se observa que existen diferencias significativas entre hombres y mujeres respecto a la actividad emprendedora. Según informe del GEM Colombia (2012), existe mayor disparidad de género en Colombia, en comparación con el promedio reportado por países de LAC, respecto a la tasa de actividad emprendedora. De acuerdo con lo destacado previamente, para controlar posibles diferencias dadas por el género del propietario-gerente, se ha introducido una variable de control binaria que asume el valor de uno (1) cuando el 
propietario-gerente es hombre, y un valor nulo (0) cuando se tiene propietario-gerente mujer.

Edad: al igual que el género, la edad puede tener influencia sobre los resultados cuando tenemos al individuo como objeto de análisis (Fuentes García y Sánchez Cañizares, 2010). En el caso de Colombia se observa que en determinados grupos etarios existe mayor proporción de emprendedores, así como diferencias respecto a características de edad entre los emprendedores establecidos (GEM, 2012). Así, se introduce una variable de control binaria para la edad, considerando dos grupos etarios: la variable asume el valor de uno (1) cuando el propietarios-gerente tiene entre 18 y 34 años, y valor nulo (0) cuando el propietario-gerente tiene entre 35 y 64 años de edad. Según clasificación del GEM, en la franja de 18 a 34 años de edad se encuentra la mayor proporción de emprendedores por oportunidad. Se espera que entre los emprendedores por oportunidad también se identifique un mayor índice de propietarios-gerentes comprometidos con aspectos de innovación.

\section{Análisis y resultados}

Previo al análisis de regresión se han observado posibles correlaciones entre las variables del modelo. Se pudo observar que la magnitud de la correlación entre las variables independientes en el modelo de regresión logística binaria no representa problemas de multicolinealidad (Hair, Anderson, Tatham y Black, 1998). Por otro lado, se esperaba una correlación entre las variables dependientes iProdServ y TechDisp que son las variables relacionadas con la innovación. El cuadro 3 presenta la correlación entre las variables de los modelos de regresión binaria utilizados para verificar las Hipótesis 1 y 2 .

\subsection{Propensión a exportar y compromiso del propietario-gerente con la innovación}

Con el ánimo de explorar si la propensión a exportar puede explicar el compromiso percibido de los propietarios-gerentes hacia aspectos de innovación, se desarrolla un procedimiento que permite estimar el cambio en la probabilidad del evento es-

Cuadro 3. Correlación entre variables

\begin{tabular}{|c|c|c|c|c|c|c|c|c|c|c|c|c|}
\hline & \multicolumn{6}{|c|}{2010} & \multicolumn{6}{|c|}{2011} \\
\hline Variables & (1) & (2) & (3) & (4) & (5) & (6) & (1) & (2) & (3) & (4) & (5) & (6) \\
\hline (1)PropExp. & 1,00 & & & & & & 1,00 & & & & & \\
\hline (2)IntensExp. & $0,91^{\text {t*t }}$ & 1,00 & & & & & $0,88^{* * *}$ & 1,00 & & & & \\
\hline (3)iProdServ. & $0,24^{\text {t*t* }}$ & $0,21^{* * *}$ & 1,00 & & & & $0,12 *$ & $0,11^{1 *}$ & 1,00 & & & \\
\hline (4)TechDisp. & $0,09^{* *}$ & $0,09^{* *}$ & $0,17^{4+x}$ & 1,00 & & & $0,12^{* *}$ & $0,09^{*}$ & $0,14^{* * t}$ & 1,00 & & \\
\hline (5)Edad & $0,09^{* *}$ & $0,12^{* *}$ & $0,09^{* *}$ & $0,14^{*+*}$ & 1,00 & & $0,08^{*}$ & $0,07^{*}$ & $0,09^{*}$ & $0,10^{* *}$ & 1,00 & \\
\hline (6)Genero & 0,01 & 0,01 & $-0,04$ & $-0,04$ & $-0,01$ & 1,00 & 0,04 & 0,01 & $-0,09^{*}$ & $-0,05$ & $0,07^{*}$ & 1,00 \\
\hline
\end{tabular}

Nota: ${ }^{*} p \leq 0,10 ;{ }^{* *} p \leq 0,05 ;{ }^{* * *} p \leq 0,01$. Fuente: elaboración propia. 
perado. Dado que la variable dependiente es binaria, el modelo de regresión logística con el análisis de los efectos marginales es el más apropiado. La regresión logística es un análisis que predice la probabilidad de ocurrencia de un evento (Hair et al., 1998), pero los parámetros estimados desde el modelo Logit sólo indican la dirección de los efectos de cada variable independiente sobre la probabilidad de respuesta. Si queremos un mejor entendimiento de los resultados es necesario calcular el efecto marginal, el cual es el cambio en la probabilidad del evento de interés como resultado de un cambio específico en una variable independiente manteniendo el resto de las variables constantes en sus medias muestrales, esto es, $\hat{\mathrm{g}}_{x}=\operatorname{Pr}(Y=1 \mid X=1)-\operatorname{Pr}(Y=1 \mid X=1)$, para el caso de variables dicotómicas. El cuadro 4 muestra los resultados de las estimaciones de la regresión logística considerando los efectos marginales.

La prueba del estadístico Chi cuadrado de los modelos completos tanto de iProdServ como de TechDisp, fue significativa e indica la existencia de una relación positiva entre el conjunto de variables independientes y las dependientes. El cuadro 4 muestra el grado de probabilidad, la significancia en el nivel correspondiente y la desviación estándar. Los valores predichos para las variables dependientes se refieren al coeficiente que predice el cambio en la probabilidad del evento de interés como resultado de un cambio específico en una variable independiente, y la interpretación es entonces análoga a la de la regresión lineal (Hair et al., 1998). Un coeficiente positivo implica que un incremento en esas variables representa una mayor pro- babilidad de que el propietario-gerente esté comprometido con la innovación.

Respecto a las variables de control, los resultados apuntan revelaciones interesantes con relación al género de los propietariosgerentes, así como de los diferentes grupos etarios. El género parece ser relevante a la hora de determinar el compromiso del propietario-gerente con aspectos relacionados con la innovación dentro de la organización. A partir de los coeficientes del modelo de regresión se observa una disminución de la probabilidad de que el propietario-gerente reconozca estar involucrado con la innovación cuando el propietario-gerente es hombre. En el año 2011 estos resultados fueron más robustos al ser estadísticamente significativos en ambos modelos (modelo sólo con las variables de control y modelo completo). Además, en el 2011 estos resultados se confirman en ambos casos: compromiso con la innovación en productos y servicios y en el uso de tecnologías de proceso más recientes.

Al considerar la edad como variable de control, se observa que los propietarios-gerentes entre las edades de 18 y 34 años presentan mayor probabilidad de que estén comprometidos con la innovación en las organizaciones que dirigen, frente a los propietarios-gerentes entre las edades de 35 y 64 años. En ambos años del análisis se confirma una relación positiva y significativa del grupo etario entre 18 y 34 años sobre el compromiso con la innovación en productos y servicio, así como, en el uso de tecnologías de proceso más recientes.

Respecto a la Hipótesis 1, se examinó si el cambio en la variable binaria (propensión a 
exportar si/no) aumentaba la probabilidad del propietario-gerente a reconocer estar involucrado con la innovación. Los resultados en el cuadro 4 demuestran que la propensión a exportar tiene un efecto positivo y significativo sobre el cambio en la probabilidad del evento de interés: compromiso de los propietarios-gerentes con la innovación. Tanto en el compromiso con la innovación en productos o servicios (H1a) como en el uso de tecnologías de proceso recientes $(\mathrm{H} 1 \mathrm{~b})$, la probabilidad de que el evento ocurriera es más alta en las empresas exportadoras que en las no exportadoras. Además, los resultados confirman que la propensión a exportar tiene un alto poder de explicación en los dos años analizados. Por tanto, se puede confirmar la Hipótesis 1.

\subsection{Intensidad exportadora y el compromiso del propietario-gerente con la innovación}

Para verificar el posible efecto de la intensidad exportadora sobre el compromiso con la innovación, se consideran únicamente las empresas con actividad exportadora. Dichas empresas son divididas en dos gru-

Cuadro 4. Estimaciones del Logit: cambio en la probabilidad de compromiso del propietario-gerente con la innovación según la propensión exportadora

\begin{tabular}{|c|c|c|c|c|c|c|c|c|c|}
\hline \multirow{3}{*}{ 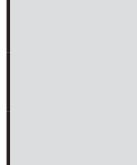 } & \multirow{3}{*}{ Control } & \multicolumn{4}{|c|}{2010} & \multicolumn{4}{|c|}{2011} \\
\hline & & \multicolumn{2}{|c|}{ iProdServ. } & \multicolumn{2}{|c|}{ TechDisp. } & \multicolumn{2}{|c|}{ iProdServ. } & \multicolumn{2}{|c|}{ TechDisp. } \\
\hline & & Modelo1 & Modelo2 & Modelo1 & Modelo2 & Modelo1 & Modelo2 & Modelo1 & Modelo2 \\
\hline Género & binaria & $\begin{array}{l}-0,051 \\
(0,032)\end{array}$ & $\begin{array}{l}-0,056^{*} \\
(0,033)\end{array}$ & $\begin{array}{l}-0,040 \\
(0,030)\end{array}$ & $\begin{array}{l}-0,041 \\
(0,030)\end{array}$ & $\begin{array}{l}-0,091^{* *} \\
(0,028)\end{array}$ & $\begin{array}{l}-0,094^{* *} \\
(0,028)\end{array}$ & $\begin{array}{l}-0,071^{*} \\
(0,037)\end{array}$ & $\begin{array}{l}-0,077^{* *} \\
(0,038)\end{array}$ \\
\hline Edad & binaria & $\begin{array}{l}0,105^{* *} \\
(0,032)\end{array}$ & $\begin{array}{l}0,086^{* *} \\
(0,034)\end{array}$ & $\begin{array}{l}0,140^{* * *} \\
(0,031)\end{array}$ & $\begin{array}{l}0,132^{\star \star *} \\
(0,031)\end{array}$ & $\begin{array}{l}0,090^{* *} \\
(0,028)\end{array}$ & $\begin{array}{l}0,082^{* *} \\
(0,028)\end{array}$ & $\begin{array}{l}0,114^{* *} \\
(0,036)\end{array}$ & $\begin{array}{l}0,104^{* *} \\
(0,037)\end{array}$ \\
\hline \multirow[t]{2}{*}{ Hipótesis } & Comp. Exportador & & & & & & & & \\
\hline & Propensión Exportar & & $\begin{array}{l}0,243^{* * *} \\
(0,031)\end{array}$ & & $\begin{array}{l}0,080^{* *} \\
(0,029)\end{array}$ & & $\begin{array}{l}0,100^{* *} \\
(0,030)\end{array}$ & & $\begin{array}{l}0,119^{* * *} \\
(0,034)\end{array}$ \\
\hline \multicolumn{10}{|c|}{ Ajuste Modelo } \\
\hline & $N$ & 1050 & 1050 & 1050 & 1050 & 782 & 782 & 782 & 782 \\
\hline & LR Chi2(df) & $12,83(2)$ & $71,88(3)$ & $22,22(2)$ & $29,50(3)$ & $16,92(2)$ & $28,41(3)$ & $12,43(2)$ & $24,22(3)$ \\
\hline & Prob>Chi2 & 0,0016 & 0,0000 & 0,0000 & 0,0000 & 0,0002 & 0,0000 & 0,0020 & 0,0000 \\
\hline & Pseudo R2 & 0,0089 & 0,0497 & 0,0170 & 0,0226 & 0,0209 & 0,0351 & 0,0122 & 0,0238 \\
\hline & Predicción correcta & & $59,71 \%$ & & $66,19 \%$ & & $78,77 \%$ & & $64,58 \%$ \\
\hline
\end{tabular}

Nota: ${ }^{*} p \leq 0,10 ;{ }^{* *} p \leq 0,05 ;{ }^{* * *} p \leq 0,01$. Los números entre paréntesis son los errores estándar. El efecto marginal representa el cambio en la probabilidad como resultado de un cambio en la variable independiente. El efecto marginal del término de interacción para el cambio en dos variables $\left(X_{2}, X_{3}\right)$ es estimado por $g_{x 2, x 3}=\frac{D^{2} F(X, b)}{D x_{2} D x_{3}}$. Fuente: elaboración propia. 
pos, de acuerdo con el grado de intensidad de sus ventas al exterior. El primer grupo está conformado por empresas que exportan hasta el 25\% del total de sus ventas y el segundo grupo por empresas que exportan un $26 \%$ o más. La Hipótesis 2 examina si el cambio en la variable binaria (intensidad exportadora media/alta o baja) aumenta la probabilidad de la capacidad innovadora de los propietarios-gerentes respecto a la innovación en productos o servicios y el uso de tecnologías de proceso recientes.

Respecto a las variables de control, se repite una tendencia ya observada en el modelo de regresión utilizado para la Hipótesis 1. Primero, considerando la variable género, en el año 2011 se observa una disminución de la probabilidad del propietario-gerente de reconocer estar involucrado con la innovación en productos o servicios cuando la variable independiente asume el valor de uno (1). Es decir, al considerar únicamente las empresas exportadoras, cuando los propietariosgerentes son mujeres hay más compromiso con la innovación en productos y servicios. Segundo, considerando la variable edad, al igual que en el modelo anterior en ambos años, se confirma una relación positiva y significativa del grupo etario entre 18 y 34 años respecto al compromiso con la innovación en productos y servicio, así como en el uso de tecnologías de proceso más recientes.

Finalmente, respecto a la Hipótesis 2, se examinó si el cambio en la variable binaria (intensidad exportadora media/alta o baja) aumentaba la probabilidad del propietariogerente de reconocer estar involucrado con la innovación. De acuerdo con los resultados expuestos en el cuadro 5, no fue posible confirmar que un cambio en la variable independiente tenga un efecto positivo y significativo sobre el compromiso de los propietariosgerentes de la muestra con la innovación. Al considerar a penas las empresas exportadoras, se esperaban diferencias significativas entre los grupos de empresas con mayor y con menor porcentaje de ventas al exterior, sin embargo, no ha sido posible confirmar estadísticamente la Hipótesis 2 del estudio.

\section{Discusión y conclusiones}

El propósito de esta investigación era indagar la probabilidad de la existencia de una relación positiva entre el comportamiento exportador de la empresa y el compromiso de los propietarios-gerentes con la innovación teniendo en cuenta el enfoque de aprendizaje.

Se hizo particular énfasis en la innovación de los productos o servicios, el uso de tecnologías de proceso recientes, el hecho de vender en el exterior y el porcentaje de dichas ventas. En general, los hallazgos indican que el compromiso de los propietarios-gerentes con la innovación de las organizaciones, en los años 2010 y 2011 en Colombia, ha sido mayor en las empresas con propensión exportadora. Debido a la imposibilidad de tratar la información disponible a través de un análisis longitudinal, la confirmación de los resultados en ambos años es importante pues refuerza una idea de tendencia y respalda las conclusiones que se desprenden de los hallazgos de esta investigación.

Colombia, a ejemplo de LAC, es un país que posee considerable actividad emprendedora 
Cuadro 5. Estimaciones del Logit: cambio en la probabilidad de compromiso del propietario-gerente con la innovación según la intensidad exportadora

\begin{tabular}{|c|c|c|c|c|c|c|c|c|c|}
\hline \multirow{3}{*}{ 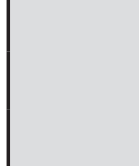 } & \multirow{3}{*}{ Control } & \multicolumn{4}{|c|}{2010} & \multicolumn{4}{|c|}{2011} \\
\hline & & \multicolumn{2}{|c|}{ iProdServ } & \multicolumn{2}{|c|}{ TechDisp } & \multicolumn{2}{|c|}{ iProdServ } & \multicolumn{2}{|c|}{ TechDisp } \\
\hline & & Modelo1 & Modelo2 & Modelo1 & Modelo2 & Modelo1 & Modelo2 & Modelo1 & Modelo2 \\
\hline Género & binaria & $\begin{array}{c}0,003 \\
(0,051)\end{array}$ & $\begin{array}{c}0,004 \\
(0,051)\end{array}$ & $\begin{array}{c}0,022 \\
(0,050)\end{array}$ & $\begin{array}{c}0,021 \\
(0,050)\end{array}$ & $\begin{array}{l}-0,081^{* *} \\
(0,033)\end{array}$ & $\begin{array}{l}-0,081^{* *} \\
(0,033)\end{array}$ & $\begin{array}{l}-0,062 \\
(0,050)\end{array}$ & $\begin{array}{l}-0,065 \\
(0,051)\end{array}$ \\
\hline Edad & binaria & $\begin{array}{l}0,091^{*} \\
(0,049)\end{array}$ & $\begin{array}{l}0,100^{* *} \\
(0,049)\end{array}$ & $\begin{array}{l}0,146^{\star *} \\
(0,049)\end{array}$ & $\begin{array}{l}0,144^{* *} \\
(0,050)\end{array}$ & $\begin{array}{l}0,091^{* *} \\
(0,033)\end{array}$ & $\begin{array}{l}0,091^{* *} \\
(0,033)\end{array}$ & $\begin{array}{c}0,084^{*} \\
(0,047)\end{array}$ & $\begin{array}{l}0,085^{*} \\
(0,047)\end{array}$ \\
\hline \multirow[t]{2}{*}{ Hipótesis } & Comp. Exportador & & & & & & & & \\
\hline & $\begin{array}{l}\text { Intensidad Exporta- } \\
\text { dora }\end{array}$ & & $\begin{array}{l}-0,075 \\
(0,059)\end{array}$ & & $\begin{array}{c}0,023 \\
(0,058)\end{array}$ & & $\begin{array}{c}0,006 \\
(0,041)\end{array}$ & & $\begin{array}{l}-0,054 \\
(0,056)\end{array}$ \\
\hline \multicolumn{2}{|c|}{ Ajuste Modelo } & & & & & & & & \\
\hline & $\mathrm{N}$ & 408 & 408 & 408 & 408 & 465 & 465 & 465 & 465 \\
\hline & LR Chi2(df) & $3,39(2)$ & $4,99(3)$ & $8,91(2)$ & $9,07(3)$ & $10,94(2)$ & $10,97(3)$ & $4,26(2)$ & $5,21(3)$ \\
\hline & Prob>Chi2 & 0,1835 & 0,1724 & 0,0116 & 0,0284 & 0,0042 & 0,0119 & 0,1191 & 0,1571 \\
\hline & Pseudo R2 & 0,0062 & 0,0091 & 0,0166 & 0,0169 & 0,0258 & 0,0259 & 0,0068 & 0,0083 \\
\hline & Predicción correcta & & $39,46 \%$ & & $62,99 \%$ & & $60,65 \%$ & & $59,57 \%$ \\
\hline
\end{tabular}

Nota: ${ }^{*} p \leq 0,10 ;{ }^{* *} p \leq 0,05 ;{ }^{* * *} p \leq 0,01$. Los números entre paréntesis son los errores estándar. El efecto marginal representa el cambio en la probabilidad como resultado de un cambio en la variable independiente. El efecto marginal del término de interacción para el cambio en dos variables $\left(X_{2}, X_{3}\right)$ es estimado por $g_{x 2, x 3}=\frac{D^{2} F(X, b)}{D x_{2} D x_{3}}$.

Fuente: elaboración propia.

pero con poca intensidad innovadora. Las investigaciones existentes demuestran que el crecimiento, e incluso, la supervivencia de las empresas, dependen de la productividad y la innovación, que actúan como facilitadores al acceso a nuevos mercados. Por otro lado, considerando la información utilizada en este estudio, apenas una tercera parte de los propietarios-gerentes encuestados en ambos años, han afirmado su compromiso con el uso de tecnologías de proceso recientes. Este tipo de evidencia ayuda a entender el alto índice de mortalidad de las empresas que no logran superar los tres años de actividad.
Considerando la primera hipótesis del estudio, se confirma la asociación positiva entre la propensión a exportar y el incremento de la probabilidad de compromiso del propietario-gerente con la innovación. Es decir, en las empresas con propensión exportadora se incrementan la innovación percibida en los productos o servicios así como el uso de tecnologías de proceso más recientes. Este superior compromiso hacia la innovación puede estar relacionado con la habilidad de la empresa de obtener nuevos conocimientos en los mercados a los que está exportando. Así, las empresas exportadoras 
tienden a incrementar su capacidad innovadora absorbiendo el conocimiento y las ideas de otros países. Por tanto, nuestros hallazgos son consistentes y soportan resultados que indican la existencia del aprendizaje a través de la presencia en mercados internacionales (Kafouros et al., 2008; Monreal-Pérez et al., 2012; Salomon y Jin, 2008; Salomon y Shaver, 2005).

Sin embargo, con respecto a la segunda hipótesis del estudio, no ha sido posible confirmar que el compromiso del propietario-gerente con la innovación sea mayor en aquellas empresas con intensidad exportadora media/ alta. Dicho resultado refuerza la idea que todavía son necesarios estudios acerca de los antecedentes y fuerzas motoras de la innovación. Se entiende que parte de los motivos por los cuales no ha sido posible confirmar la Hipótesis 2 tienen relación directa con algunas limitantes de medidas y análisis como se discute más adelante en el apartado 4.2.

En conclusión, ¿el comportamiento exportador de la organización hace que el propietario-gerente tenga un mayor compromiso con la innovación? En consonancia con el enfoque de aprendizaje mediante la actividad exportadora, éste es un fenómeno posible. Aunque se necesitan más evidencias que examinen el efecto del comportamiento exportador sobre el compromiso de los directivos y la capacidad de innovación de las empresas, nuestros hallazgos refuerzan algunos estudios que empíricamente comprueban la existencia de efectos positivos en esta relación (ej. Filipescu et al., 2009; Molero, 1998; Monreal-Pérez et al., 2012; Podmetina, Smirnova, Vaatanen y Torkkeli,
2009; Salomon y Shaver, 2005). Estos resultados podrían explicarse por la necesidad que tienen las empresas de innovar para acceder y permanecer en mercados internacionales.

Considerando que el nuevo panorama económico requiere de una combinación de la iniciativa empresarial, la innovación y la internacionalización, los resultados de este estudio en el contexto de Colombia sugieren que la actividad exportadora es más que una actividad para alcanzar nuevos mercados y aumentar el volumen de ventas (Hagen, Denicolai y Zucchella, 2014). La actividad exportadora puede actuar como fuente de información que, absorbida como aprendizaje, podría generar innovación.

\subsection{Contribuciones del estudio}

Al considerar dos años consecutivos, nuestros hallazgos pueden indicar una tendencia sobre las relaciones propuestas en el estudio. Por tanto, podemos afirmar que este artículo contribuye a la literatura académica y presenta algunas implicaciones en la práctica. Para los académicos, este estudio se adhiere a la corriente de investigación que explica los antecedentes de la innovación. El estudio aporta una perspectiva diferente, proveyendo evidencia a partir del propietario-gerente de la organización y su compromiso con la capacidad innovadora dentro de la organización.

En resumen, la habilidad del propietariogerente para asumir el rol de responsable sobre decisiones que lleven a la innovación en productos o servicios es mayor cuando la organización está presente en otros mercados internacionales a través de las exportaciones. 
Además, el uso de tecnologías de procesos con menos de cinco años también es más frecuente en las empresas con experiencia exportadora. Así, utilizando la capacidad y el compromiso con la innovación como un reflejo del aprendizaje externo, se concluye que las empresas pueden acceder estratégicamente a conocimientos en el extranjero a través de la actividad exportadora.

La propuesta de investigación del estudio trata de contribuir en dimensiones aún subexplotadas como el efecto del comportamiento exportador sobre aspectos relacionados con la innovación bajo un acercamiento a la capacidad de absorción del conocimiento adquirido en el exterior. Por tanto, las contribuciones teóricas de este estudio al enfoque de aprendizaje yacen en la extensión de la investigación sobre la capacidad innovadora con un énfasis en la propensión a exportar. También vale destacar la aportación al conocimiento acerca de la innovación dentro de la organización considerando el propietariogerente como el objeto de estudio.

Para los propietarios-gerentes de empresas, los resultados de este estudio sugieren que exportar es más que una decisión de incrementar las ventas y alcanzar otros mercados. Mientras aprenden por su experiencia en mercados foráneos, las empresas a través de sus propietarios-gerentes, también incrementan su capacidad para innovar.

\subsection{Limitaciones y futuras líneas de investigación}

Este artículo está sujeto a ciertas limitaciones que tipifican la investigación comportamen- tal y sugerimos precaución al interpretar sus resultados. Dado que la información considerada para el análisis ha sido secundaria, no se ha podido contar con ciertas variables y/o medir las variables utilizadas en los modelos de la manera más adecuada. Así, una serie de limitantes pueden ayudar a explicar por qué no fue posible confirmar la Hipótesis 2. Primero, el volumen de las exportaciones no necesariamente indica que la empresa exporta a varios países. En este caso podría haber una concentración de las exportaciones a un único país lo que no se puede verificar con la base de datos disponible. Siendo así, esto podría ser uno de los motivos por los cuales no se encontraron diferencias significativas, respecto al aprendizaje mediante la exportación, entre los grupos de empresas con porcentaje de exportaciones medio/alto y bajo. En este sentido, futuros estudios podrían considerar diferentes escalas de la actividad exportadora como por ejemplo el alcance de las exportaciones. Es decir, considerar una dimensión en que conste el número de países en los que la empresa tiene actividades de exportación.

Segundo, la proporción o volumen de operaciones de exportación a otros mercados puede variar de acuerdo con la industria y el tiempo de internacionalización, y como tal, puede no ser indicativo de su éxito global durante las primeras etapas de internacionalización a través de las exportaciones. Futuras investigaciones deberían considerar el comportamiento exportador en términos de escala para observar como el aprendizaje afecta la propensión a innovar. En este caso, considerar la industria y el tiempo que la empresa lleva realizando exportaciones podría 
ayudar a entender mejor este fenómeno. Una empresa que ha empezado sus actividades internacionales desde hace muchos años o incluso décadas, puede generar mayor volumen de negocios en mercados extranjeros que empresas que recién empiezan a exportar (Torkkeli et al., 2012, p. 38).

Tercero, con respecto al bajo poder explicativo del modelo, a juzgar por el índice obtenido del Pseudo R2, otras variables pueden estar interviniendo en la relación estudiada. Así, futuros estudios podrían incluir en el análisis aspectos como el uso de las redes por parte de las empresas y su rol en la unión entre la internacionalización y la innovación, ya que las conexiones interpersonales desarrolladas en un nuevo mercado, acopladas a las decisiones estratégicas en el país de origen, pueden fomentar los procesos de aprendizaje estimulando así la innovación. De la misma manera, otras variables del campo del emprendimiento como la proactividad y la propensión a asumir riesgos más allá de las fronteras nacionales, podrían ayudar en la clarificación de los vínculos entre el comportamiento exportador y la innovación.

Cuarto, es importante destacar la existencia de muchas más investigaciones observando un solo lado de la relación (exportación determina la innovación o la innovación determina la exportación). Así, futuros estudios que consideren los efectos simultáneos entre la innovación y el comportamiento exportador en su sentido más amplio (propensión, intensidad, alcance global, etc.), serán de gran aporte a este campo del análisis.
Finalmente, a pesar de que el estudio considere información de dos años y los resultados indiquen una tendencia en ambos, un examen longitudinal con la misma muestra de empresas podría ser útil para entender el fenómeno. El análisis longitudinal también podría dar más información sobre los efectos a largo plazo del aprendizaje mediante la actividad exportadora sobre el compromiso de los propietarios-gerentes hacia la innovación dentro de la empresa (Damjan, Kostevc y Polanec, 2010; Salomon y Shaver, 2005). Esto podría facultarnos para clarificar la relación de causalidad entre el comportamiento exportador y la capacidad innovadora en las organizaciones.

\section{Referencias}

Acs, Z. J., and Amorós, J. E. (2008). Entrepreneurship and competitiveness dynamics in Latin America. Small Business Economics, 31 (3), 305-322.

Amorós, J., and Cristi, O. (2008). Longitudinal analysis of entrepreneurship and competitiveness dynamics in Latin America. International Entrepreneurship and Management Journal, 4 (4), 381-399.

Anand, B., and Khanna, T. (2000). Do firm learn to create value? The case of alliances. Strategic Management Journal, 21 (3), 295-315.

Autio, E., Sapienza, H., and Almeida, J. (2000). Effects of age at entry, knowledge intensity, and imitability on international growth. Academy of Management Journal, 43 (5), 909-924.

Banco Mundial (2014). Datos por países - Colombia. Recuperado el 05 de mayo de 2014, de: http://datos.bancomundial.org/pais/ colombia\#cp_wdi 
Caldera, A. (2010). Innovation and exporting: Evidence from Spanish manufacturing firms. Review of World Economics, 146 (4), 657-689.

Cassiman, B., and Golovko, E. (2011). Innovation and internationalization through exports. Journal of International Business Studies, 42 (1), 1-20.

Cassiman, B., and Martínez-Ros, E. (2007). Product innovation and exports: Evidence from Spanish manufacturing. IESE Business School, Barcelona.

Chetty, S., and Wilson, H. (2003). Collaborating with competitors to acquire resources. International Business Review, 12 (1), 61-81.

Cohen, W., and Levinthal, D. (1990). Absorptive capacity: A new perspective on learning and innovation. Administrative Science Quarterly, 35 (1), 128-152.

Damjan, J., Kostevc, C., and Polanec, S. (2010). From innovation to exporting or vice versa? The World Economy, 33 (3), 374-398.

Denicolai, S., Zucchella, A., and Strange, R. (2014). Knowledge assets and firm international performance. International Business Review, 23 (1), 55-62.

Eiriz, V., Faria, A., and Barbosa, N. (2013). Firm growth and innovation: Toward a typology of innovation strategy. Innovation: Management, Policy \& Practice, 15 (1), 97-111.

Eriksson, K., Johanson, J., Majkgard, A., and Sharma, D. (1997). Experiential knowledge and cost in the internationalization process. Journal of International Business Studies, 28 (2), 337-360.

Fernandes, A. M., and Isgut, A. E. (2005). Learningby-doing, learning-by-exporting, and productivity: Evidence from Colombia. Washington, DC: World Bank.

Filipescu, D., Rialp, A., and Rialp, J. (2009). Internationalization and technological innovation: empirical evidence on their mutual relations- hip. Advances in International Marketing, 20, 125-154.

Filipescu, D., Prashantham, S., Rialp, A., and Rialp, J. (2013). Technological innovation and exports: Unpacking their reciprocal causality. Journal of International Marketing, 21 (1), 23-38.

Fuentes García, F. J. y Sánchez Cañizares, S. M. (2010). Análisis del perfil emprendedor: una perspectiva de género. Estudios de Economía Aplicada, 28 (3), 1-28.

Gassmann, O., and Keupp, M. (2007). The competitive advantage of early and rapidly internationalising SMEs in the biotechnology industry: A knowledge-based view. Journal of World Business, 42 (3), 350-366.

Global Entrepreneurship Monitor (2012). Reporte GEM Colombia, 2011-2012.

Golovko, E., and Valentine, G. (2011). Exploring the complementarity between innovation and export for SMEs' growth. Journal of International Business Studies, 42, 362-380.

González-Pernía, J., and Peña-Legazkue, I. (2011). FDI, external knowledge and the export-oriented behaviour of Spanish early-stage entrepreneurs. Documento presentado en el $5^{\text {th }}$ Global Entrepreneurship Research Conference, 6 al 8 de octubre, Cartagena.

Hagen, B., Denicolai, S., and Zucchella, A. (2014). International entrepreneurship at the crossroads between innovation and internationalization. Journal of International Entrepreneurship, 12 (2), 111-114.

Hair, J. F., Anderson, R. E., Tatham, R. L., and Black, W. C. (1998). Multivariate data analysis, $5^{\text {th }}$ ed. London: Prentice-Hall.

Hult, G., Hurley, R., and Knight, G. (2004). Innovativeness: Its antecedents and impact on business performance. Industrial Marketing Management, 33 (5), 429-438. 
Johanson, J., and Vahlne, J. (1991). The mechanism of internationalization. International Marketing Review, 7 (4), 11-24.

Kafouros, M., Buckley, P., Sharp, J., and Wang, C. (2008). The role of internationalization in explaining innovation performance. Technovation, 28 (1), 63-74.

Katsikeas, C., Leonidou, L., and Morgan, N. (2000). Firm-level export performance assessment: review, evaluation, and development. Journal of the Academy of Marketing Science, 28 (4), 493-511.

Kirbach, M., and Schmiedeberg, C. (2008). Innovation and export performance: Adjustment and remaining differences in East and West German manufacturing. Economics of Innovation and New Technology, 17 (5), 435-457.

Lachenmaier, S., and Wobmann, L. (2006). Does innovation cause exports? Evidence from exogenous innovation impulses and obstacles using German micro data. Oxford Economic Paper, 58, 317-350.

Lane, P., and Lubatkin, M. (1998). Relative absorptive capacity and interorganizational learning. Strategic Management Journal, 19 (5), 461-477.

Lederman, D., Messina, J., Pienknagura, S. y Rigolini, J. (2014). El emprendimiento en América Latina: muchas empresas y poca innovación. Washington DC: Banco Mundial.

López Rodríguez, J., and García Rodríguez, R. (2005). Technology and export behaviour: A resource-based view approach. International Business Review, 14 (5), 539-557.

Molero, J. (1998). Patterns of internationalization of Spanish innovatory firms. Research Policy, 27 (5), 541-558.

Monreal-Pérez, J., Aragón-Sánchez, A., and Sánchez-Marín, G. (2012). A longitudinal study of the relationship between export activity and innovation in the Spanish firms: The modera- ting role of productivity. International Business Review, 21, 862-877.

Ocasio, W. (1997). Towards an attention-based view of the firm. Strategic Management Journal, 18 (S1), 187-206.

Organización para la Cooperación y el Desarrollo Económico, OCDE (2013). Estudios económicos. Colombia- Evaluación económica. Recuperado el 10 de mayo de 2014 de: http:// www.oecd.org/eco/surveys/Colombia_Overview_ESP\%20NEW.pdf

Pan, Y., and Chi, P. S. K. (1999). Financial performance and survival of multinational corporations in China. Strategic Management Journal, 20 (4), 359-374.

Podmetina, D., Smirnova, M., Vaatanen, J., and Torkkeli, M. (2009). Innovativeness and international operations: Cause of Russian R\&D companies. International Journal of Innovation Management, 13, 295-317.

Prashantham, S. (2005). Toward a knowledge-based conceptualization of internationalization. Journal of International Entrepreneurship, 3, 37-53. Reynolds, P., Bosma, N., Autio, E., Hunt, S., De Bono, N., Servais, I., López-García, P., and Chin, N. (2005). Global entrepreneurship monitor: Data collection design and implementation 1998-2003. Small Business Economics, 24 (3), 205-231.

Sapienza, H., De Clercq, D., and Sandberg, W. (2005). Antecedents of international and domestic learning effort. Journal of Business Venturing, 20 (4), 437-457.

Salomon, R., and Jin, B. (2008). Does knowledge spill to leaders or laggards? Exploring industry heterogeneity in learning by exporting. Journal of International Business Studies, 39 (1), 132-150.

Salomon, R., and Shaver, J. (2005). Learning-byexporting: new insights from examining firm 
innovation. Journal of Economics and Management Strategy, 14 (2), 431-461.

Torkkeli, L., Puumalainen, K., Saarenketo, S., and Kuivalainen, O. (2012). The effect of network competence and environmental hostility on the internationalization of SMEs. Journal of International Entrepreneurship, 10 (1), 25-49. Varela, R., Moreno, J., and Soler, J. (2013). Global Entrepreneurship Monitor - GEM Caribbean:
Colombian National Report 2012. Cali: Universidad ICESI.

Wakelin, K. (1998). Innovation and export behavior at the firm level. Research Policy, 26 (7), 829-841.

Zahra, S., and George, G. (2002). Absorptive capacity: A review, reconceptualization, and extension. Academy of Management Review, 27 (2), 185-203. 
